\title{
Preface
}

\section{The Changing Landscape of Pancreatic Pathology}

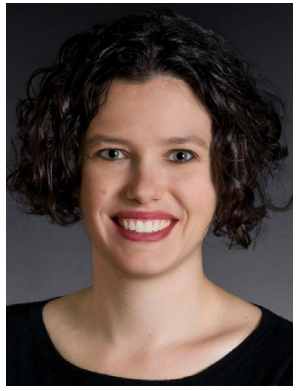

Laura D. Wood, MD, PhD

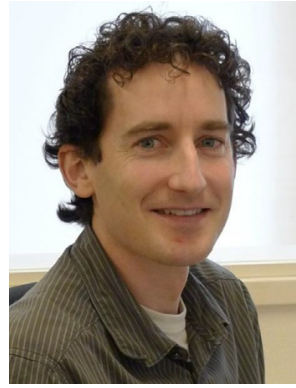

Lodewijk A.A. Brosens, MD, PhD Editors
The practice of pancreatic pathology has evolved significantly in recent years. In this issue, we provide an update into the major diagnostic entities in the pancreas, including both neoplastic and nonneoplastic diseases. In addition, we provide correlations with other specialties of relevance to the practicing pathologist. We first focus on gross dissection of pancreatic resection specimens, as this process lays the groundwork for accurate diagnosis. We then discuss the process that resulted in the gross specimen by highlighting advances in surgery that are relevant to the practicing pathologist. Then, we turn to the tumors of the pancreas, which represent the entities encountered most commonly in practice-these include pancreatic adenocarcinoma (and variants), precursor lesions, nonductal cancers, and neuroendocrine tumors. Next, we cover benign pancreatic lesions, including pancreatitis and benign masses. We then focus on other pathologic analyses that are commonly used on the pancreas, including cytopathology and cyst fluid analysis. Finally, we highlight the molecular genetics of pancreatic neoplasms, a field that has advanced greatly in recent years, and discuss genetic syndromes with pancreatic manifestations. This broad update is intended for practicing pathologists in order to capture the critical advances in recent years.

Laura D. Wood, MD, PhD Sol Goldman Pancreatic Cancer Research Center Johns Hopkins University School of Medicine

CRB2 Room 345

1550 Orleans Street Baltimore, MD 21231, USA

Lodewijk A.A. Brosens, MD, PhD Department of Pathology (H04-312)

University Medical Center Utrecht

Heidelberglaan 100

3584 CX Utrecht, The Netherlands

E-mail addresses:

Idwood@jhmi.edu (L.D. Wood)

I.a.a.brosens@umcutrecht.nl (L.A.A. Brosens) 